\title{
A giant panurinary stone revealed by pyonephrosis and acute renal failure
}

\author{
Yassine El Abiad ${ }^{* 1}$, Mohammed Bellahcen ${ }^{2}$, Bouzid Balla ${ }^{1}$, Youness Dehayni ${ }^{1}$, Abdelghani Ammani ${ }^{1}$, Abdelmounaim \\ Qarro ${ }^{1}$, Mohammed Alami ${ }^{1}$ \\ ${ }^{1}$ Urology Department, My Ismail Military Hospital, Meknes, Morocco \\ ${ }^{2}$ Med V University School of Medecine, Rabat, Morocco
}

Received: February 21, 2016

Accepted: May 23, 2016

Online Published: June 12, 2016

DOI: $10.5430 /$ css.v2n3p15

URL: http://dx.doi.org/10.5430/css.v2n3p15

\begin{abstract}
Urinary stones are a common problem with increasing incidence, but giant ones leading to severe complications are rare nowadays. We report here a historic case of panurinary stone filling almost all urinary cavities and incidentally discovered by a life-threatening sepsis and acute renal dysfunction.
\end{abstract}

Key Words: Urolithiasis, Renal stone, Bladder stone, Pyonephrosis, Renal dysfunction

\section{INTRODUCTION}

Urolithiasis is a common disease with increasing incidence, probably due to modern lifestyle. The lifetime prevalence of kidney stone disease is estimated at $1 \%$ to $15 \%$, with the probability of having a stone varying according to age, gender, race, and geographic location. ${ }^{[1]}$ Bladder calculi account for $5 \%$ of all urinary calculi, and can either complicate renal ones or be secondary to infravesical obstruction. ${ }^{[2]}$ Although it is a benign condition, urolithiasis may be responsible of several complications such as renal colic, infection, acute urinary obstruction, renal damage and long-term sequelae of stone recurrence.

Herein, we report a case of complicated urolithiasis with significant stone burden present in the bladder and both kidneys, and its step-by-step management using conventional techniques.

\section{Case presentation}

A 53-year-old man with unremarkable medical history presented to the emergency department of our hospital after
2 days of bilateral flank pain, fever and dysuria. Physical examination found a lethargique and dehydrated patient with sepsis criteria; tachycardia at 110 beats per minutes, fever at $39.5^{\circ} \mathrm{C}$ and polypnea, but he was hemodynamically stable. He also had an abdominal distension, sensitive flanks and a hard palpable mass of the suprapubic area. Digital rectal examination was normal. Laboratory tests results showed a positive urine culture for Escherichia coli, an acute kidney injury (AKI) with high blood urea and creatinine values at $2.1 \mathrm{~g} / \mathrm{L}$ (normal range $0.10-0.50$ ) and $70.5 \mathrm{mg} / \mathrm{L}$ (normal range 7-13) respectively, and a hyperleucocytosis at $17,000 / \mu 1$.

We performed an abdominal radiography (see Figure 1) which showed three giant stones at the projection of kidneys and bladder areas. The clinical suspicions of obstructive pyonephrosis were confirmed by an ultrasound (US) scan which revealed dilated renal cavities with echogenic content alongside massive stones of both kidneys and the bladder. A non-contrast computed tomographic (CT) imaging of the abdomen and pelvis was also performed (see Figures 2 and

\footnotetext{
*Correspondence: Yassine El Abiad; Email: yassineelabiad@gmail.com; Address: Urology Department, My Ismail Military Hospital, Meknes, Morocco.
} 
3 ) and showed the huge bilateral renal stones measuring approximately $5 \mathrm{~cm} \times 4.5 \mathrm{~cm} \times 2.6 \mathrm{~cm}$ each and completely filling renal pelvis, together with a giant bladder stone of a nearly $8 \mathrm{~cm} \times 6 \mathrm{~cm} \times 5.8 \mathrm{~cm}$ almost occupying the intravesical cavity. The patient was urgently given intravenous (IV) hydration with saline serum, and antibiotics. Initially, we intended to treat the patient conservatively with indwelling ureteral stents but the presence of a huge bladder stone filling completely the intravesical cavity made it impossible for the operator to identify ureteral orifices. Therefore, we carried out US-guided bilateral percutanuous nephrostomy which were very effective in draining frank pus from renal cavities.

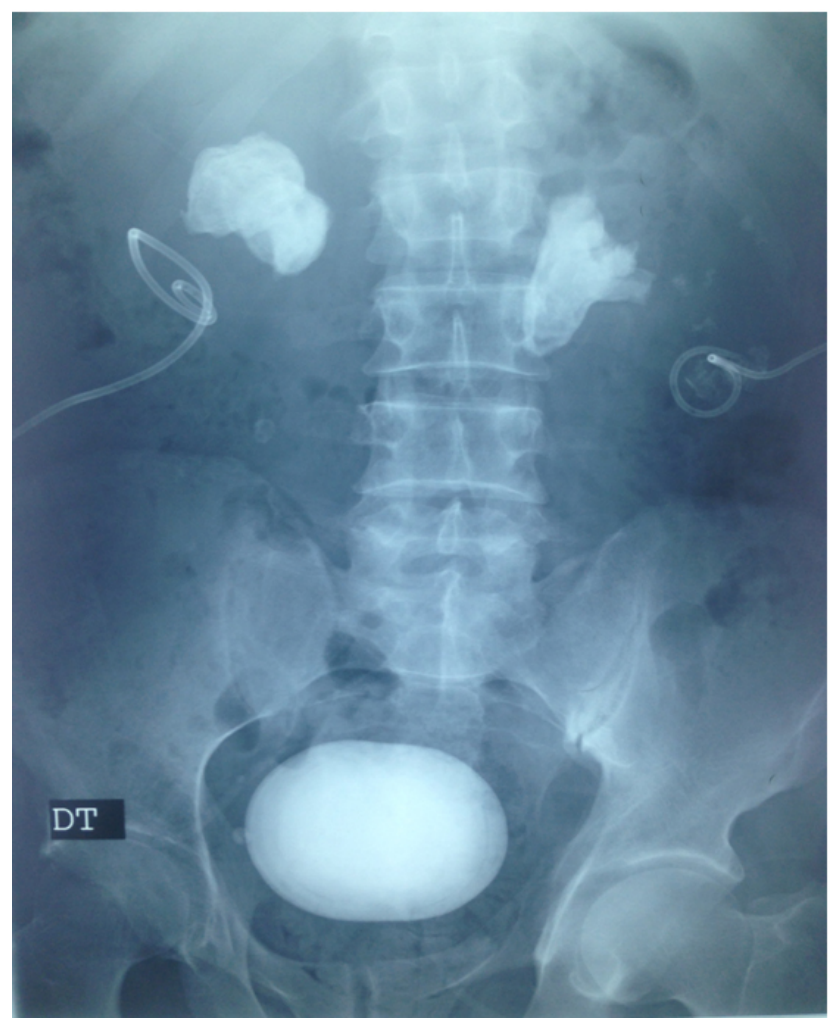

Figure 1. The plain kidneys, ureters, and bladder $X$-ray revealing three giant urinary stones with in-place bilateral nephrostomy tubes

After the patient's clinical state had improved, a cystolithotomy was performed 2 weeks later and a stone weighing more than $0.30 \mathrm{~kg}$ was extracted as one complete piece (see Figure 4). He is now scheduled for bilateral percutaneous nephrolithotomy (PCNL) that we entend to perform in two sessions and eventually complete with extracorporeal shock wave lithitripsy (ESWL) to clean renal cavities. The patient had made an uneventful recovery with slight improvement of renal function. Stone analysis found a calcium oxalate composition, whereas basic etiologic investigation revealed no metabolic abnormalities.

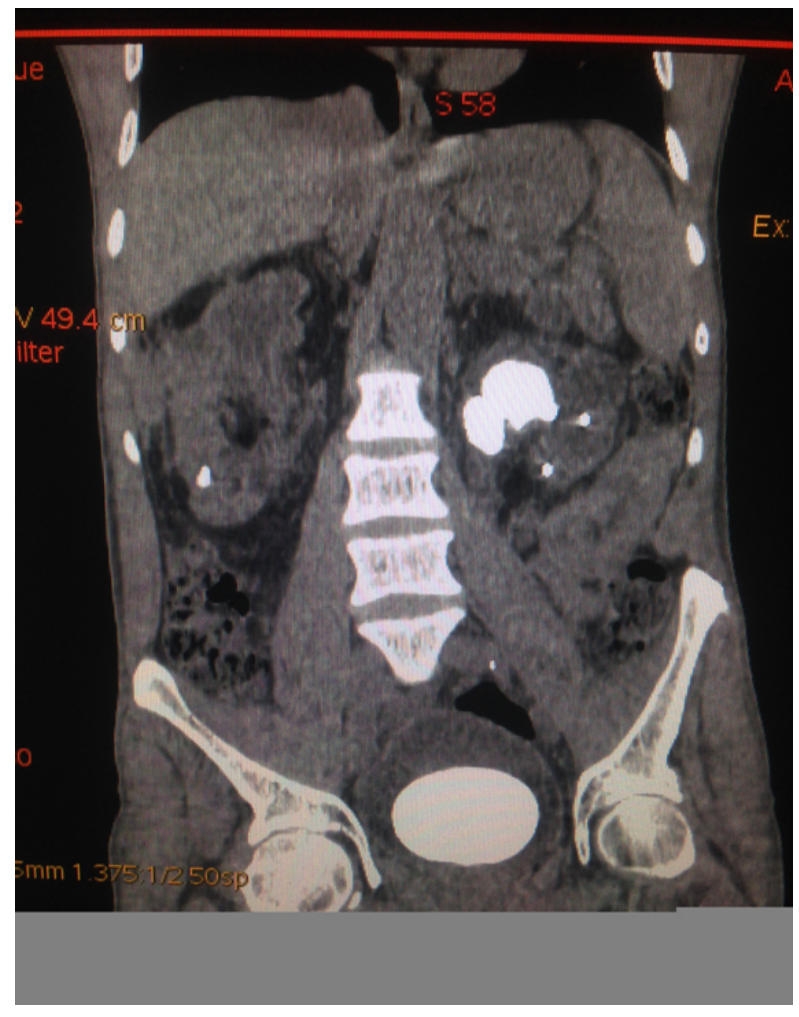

Figure 2. Non-contrast CT coronal view of the abdomen and pelvis showing the presence of bilateral renal stones together with a giant bladder calculus of a nearly $8 \mathrm{~cm} \times$ $6 \mathrm{~cm} \times 5.8 \mathrm{~cm}$ occupying the intravesical cavity

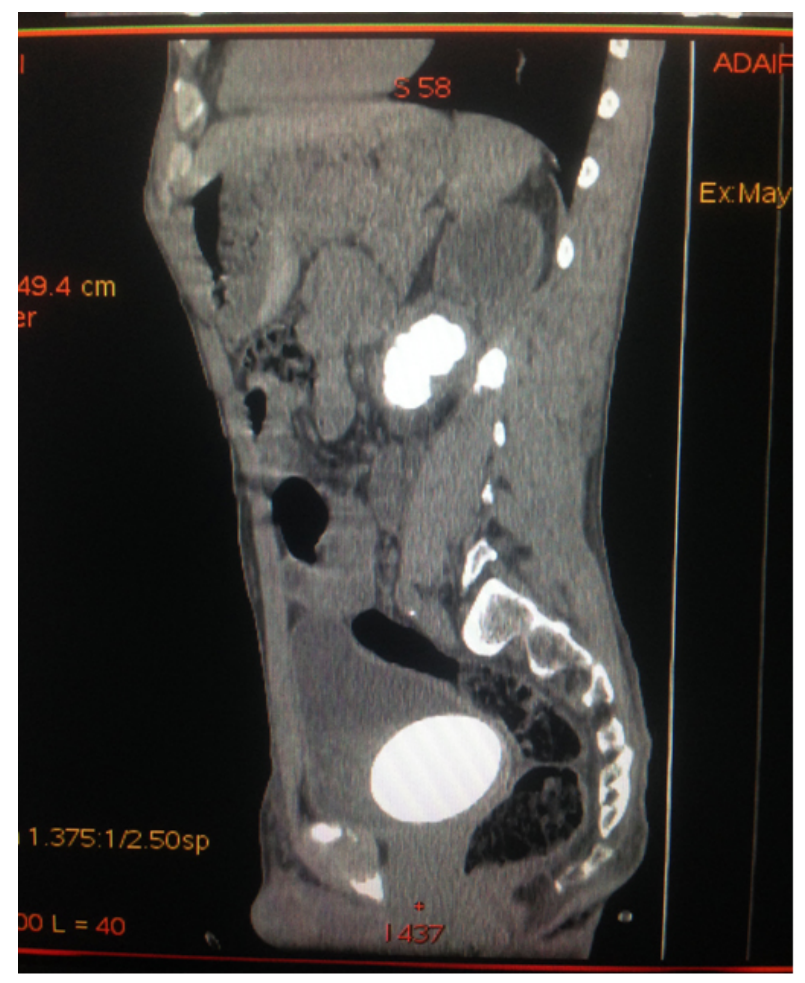

Figure 3. Non-contrast CT sagittal view of the abdomen and pelvis showing renal and bladder stones 


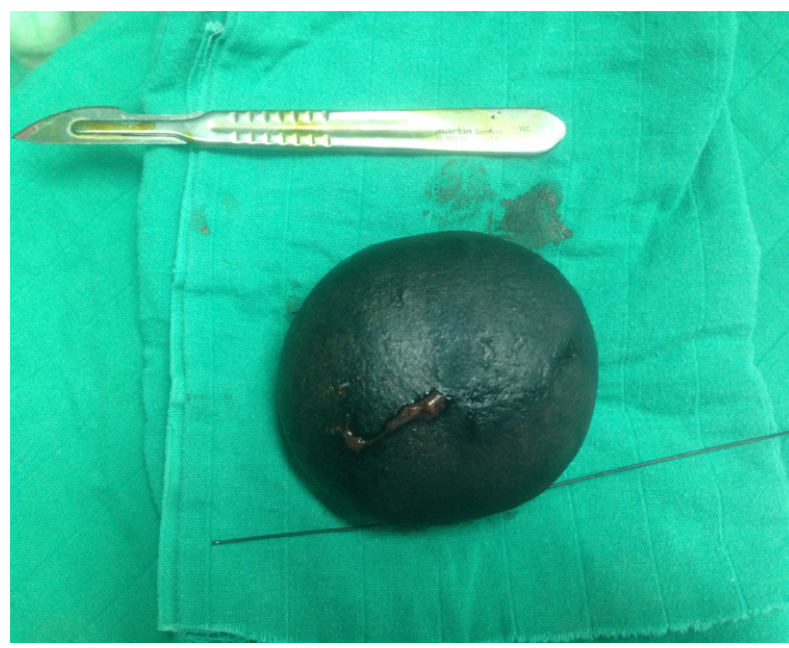

Figure 4. The huge bladder stone with eight-centimeter diameter in macroscopic view after extraction by cystolithotomy

\section{Discussion}

Urinary tract stones occur more commonly in men than women, and the risk of urolithiasis correlates with weight and body mass index in both sexes. ${ }^{[1]}$ The majority of patients with renal calculi have a small stone burden, so they can be managed early before developing severe complications. However, if left untreated, larger calculi become obstructive and infected, such as the case of our patient who had a years-long silent disease that finally lead to life-threatening pyonephosis and acute renal failure.

Bladder stones usually occur in men older than 50 years with associated bladder outlet obstruction, neurogenic voiding dysfunction, urinary tract infection or foreign bodies. ${ }^{[2,3]}$ Most bladder stones are composed of struvite calculi, but other types are also common especially calcium oxalate which indicates the presence of a probable renal stone. ${ }^{[1]}$ Since our patient had no sign of lower urinary tract obstruction and stone analysis found calcium oxalate composition, it's very likely that a renal stone had passed into the blad- der and caused the development of a giant stone. Despite the fact, the association of both kidneys massive calculi and giant bladder calculi is a rare entity in the recent urological practice. To our knowledge, our patient represents one of the largest urinary stone cases reported to date.

Pyonephrosis is a rare disease causing suppurative destruction of renal parenchyma and upper urinary tract system infections and obstructions play a role in its etiology. The accumulation of purulent exudate in the hydronephrotic collecting system and abscess formation constitute the pathophysiology of pyonephrosis. ${ }^{[4]}$ In our case, the patient developed the pyonephrosis on a background of chronic pyelonephritis due to stone related secondary obstruction. Also, the stone served as a nidus for infection and helped accelerate the process. The treatment of pyonephrosis is mainly based on renal drainage. Antibiotics have no effect unless the pus is surgically drained. ${ }^{[5]}$

Percutaneous nephrostomy and urethral catheter insertion are therefore necessary. Studies show percutaneous drainage to be a fast, trusted, and effective diagnostic and therapeutic method. ${ }^{[6]}$ As we did for our patient, all the reports have recommended open cystolithotomy for large-sized bladder stones. ${ }^{[7]}$ Fortunately, pyonephrosis in our patient was diagnosed and treated early, before the development of septic shock and death, but the destruction of renal parenchyma was so severe that the renal function improved slightly thereafter.

\section{Conclusions}

In conclusion, giant panurinary stone is rarely seen in current urological practice, and pyonephrosis with acute renal failure represent the worst clinical scenario that can arise in those patients. Early diagnosis and immediat renal drainage are necessary to avoid fatal evolution.

\section{CONFLicts OF InTEREST Disclosure}

None.

\section{REFERENCES}

[1] Wein AJ, Kavoussi LR, Novick AC, et al. Evaluation and Medical Management of Urinary Lithiasis. In: Ferrandino MN, Pietrow PK, Preminger GM eds. Campbell-walsh Urology. 10th edn. Philadelphia: Elsevier; 2012. 1320-1321 p.

[2] Schwartz BF, Stoller ML. The vesical calculus. Urol Clin North Am. 2000; 27: 333-46. http: //dx.doi.org/10.1016/S0094-0 143 (05) $70262-7$

[3] Hammad FT, Kaya M, Kazım E. Bladder calculi: did the clinical picture change? Urology. 2006; 67: 1154-8. PMid: 16765170. http://dx.doi.org/10.1016/j.urology.2005.12.038

[4] Eroglu M, Kandıralı E. Akut Pyelonefrit ve pyonefroz (Acute pyelonephritis and pyonephrosis). Turkiye Klinikleri Journal of Sur-

Published by Sciedu Press gical Medical Sciences. 2007; 3(20): 24-28.

[5] Lezin MST, Hofmann R, Stoller ML. Pyonephrosis: diagnosis and treatment. British Journal of Urology. 1992; 70(4): 360-3. http: //dx.doi.org/10.1111/j.1464-410x.1992.tb15788.x

[6] Mokhmalji H, Braun PM, Portillo FJM, et al. Percutaneous nephrostomy versus ureteral stents for diversion of hydronephrosis caused by stones: a prospective, randomized clinical trial. Journal of Urology. 2001; 165(165): 1088-92. http://dx.doi.org/10.1016/S0022 $-5347(05) 66434-8$

[7] Gallego VD, Beltran PJ, Pérez MM, et al. Giant bladder lithiasis: case report and bibliographic review. Archivos Espa-oles De Urología 2011; 64(4): 383-7. 